\title{
Connecting orbits in one-parameter families of flows
}

\author{
JAMES F. REINECK \\ Department of Mathematics, SUNY at Buffalo, Buffalo, NY 14214, USA
}

\begin{abstract}
Given a family of flows parametrized by an interval and a Morse decomposition which continues across the interval, a procedure is devised to detect connecting orbits at various parameter values. This is done by putting a small drift on the parameter space and considering the flow on the product of the phase space and the parameter interval. The Conley index and connection matrix are used to analyse the flow on the product space, then the drift is allowed to go to zero to obtain information about the original family of flows. This method can be used to detect connections between rest points of the same index for example.
\end{abstract}

\section{Introduction}

Suppose we have a one-parameter family of flows, parametrized by an open interval, and suppose that there is an isolated invariant set and Morse decomposition which continue across the interval. To obtain information about connecting orbits for different parameter values, we put an artificial flow on the parameter space. This flow has two rest points, one attracting and one repelling. The products of the Morse sets and the rest points give us a Morse decomposition of the flow on the product space. If there is a connecting orbit between two Morse sets in the product flow, and if this orbit persists as we let the artificial flow go to zero, then we can construct a 'limiting connection' in the Hausdorff topology and use this limit to obtain information about connecting orbits for parameter values between the two rest points.

This technique can be applied to understand the behaviour of families of differential equations, e.g. flows arising from ecological models [10] and travelling wave solutions to reaction-diffusion equations [8]. In the latter example the wave speed is a natural parameter.

In $\S 1$ we discuss the Hausdorff metric and in $\S 2$ we list some definitions and basic results about Morse decompositions. In $\S 3$ we discuss the properties of the limiting connection mentioned above. This limit gives us information about unstable connections, e.g. connections between two saddle points which occur at some parameter value.

In order to apply the theory of $\S 3$, we must be able to detect connections in the product flow. To do this, we introduce the Conley index and the connection matrix of Franzosa. The connection matrix is an algebraic object which gives us information about the connecting orbits in a flow. If the matrix at two parameter values is known, then these can be used to compute the matrix for the product flow. In $\$ 4$ we discuss the basic properties of the connection matrix and in $\S 5$ we discuss the use of the connection matrix in the product flow. 


\section{The Hausdorff metric}

Let $(X, d)$ be a compact metric space and let $\mathscr{F}$ denote the closed subsets of $X$. For $A, B \in \mathscr{F}$ let

$$
\begin{aligned}
& h(A, B)=\inf \{\varepsilon \mid A \text { is contained in an } \varepsilon \text {-neighbourhood of } B\}, \\
& \rho(A, B)=\max \{h(A, B), h(B, A)\} .
\end{aligned}
$$

It is well known that $\rho$ is a metric on $\mathscr{F}$, called the Hausdorff metric, and that $(\mathscr{F}, \rho)$ is a compact metric space. For $x, y \in X, A, B \in \mathscr{F}$ we have the following generalizations of the triangle inequality:

$$
\begin{aligned}
& d(x, A) \leq d(x, y)+d(y, A), \\
& d(x, A) \leq d(x, B)+\rho(B, A) .
\end{aligned}
$$

The following results are well known and easy so we omit the proofs.

Lemma 1.1. Suppose $\left\{A_{n}\right\}$ is a sequence in $\mathscr{F}, A_{n} \rightarrow A$. Then

$$
A=\left\{x \in X \mid \text { there is a sequence } x_{n} \in A_{n} \text { with } x_{n} \rightarrow x\right\} \text {. }
$$

Lemma 1.2. Suppose $\left\{A_{n}\right\}$ is a sequence in $\mathscr{F}$ with $A_{n}$ connected for each $n$. If $A_{n} \rightarrow A$, then $A$ is connected.

\section{Morse decompositions}

In this section we summarize some recent results on Morse decompositions.

Definition 2.1. A partially ordered set is a pair $(P,<)$ consisting of a finite set $P$ and a relation $<$ on $P$ satisfying:

(a) for no $p \in P$ is $p<p$;

(b) $p<q$ and $q<r$ implies $p<r$ for all $p, q, r \in P$.

Definition 2.2. Let $(P,<)$ be a partially ordered set.

(a) $I \subset P$ is an interval if $p, r \in I, q \in P, p<q<r$ implies $q \in I$. We denote the set of intervals by $\mathscr{I}(P,<)$ or $\mathscr{I}(P)$.

(b) $A \subset P$ is an attracting interval if $a \in A, p \in P, p<a$ implies $p \in A$. Let $\mathscr{A}(P)$ denote the attracting intervals.

(c) A pair of intervals $(I, J)$ is an adjacent pair of intervals if $I \cup J$ is an interval and for no $i \in I, j \in J$ is $j<i$. If $(I, J)$ is an adjacent pair, we write $I J$ instead of $I \cup J$.

Let $\Gamma$ be a Hausdorff space with a flow and let $X \subset \Gamma$ be a local flow. We assume that the reader is familiar with $\omega$ - and $\omega^{*}$-limit sets, isolated invariant sets, attractorrepeller pairs and continuation as discussed in [1] and [12]. If $S_{1}$ and $S_{2}$ are invariant sets in $X$, then the set of connections from $S_{2}$ to $S_{1}$ is the set

$$
C\left(S_{2}, S_{1}\right)=\left\{x \in X \mid \omega(x) \subset S_{1} \text { and } \omega^{*}(x) \subset S_{2}\right\} .
$$

Definition 2.3. Let $(P,<)$ be a partially ordered set, $S \subset X$ an isolated invariant set. A $(P,<)$-ordered Morse decomposition of $S$ is a collection $\left\{M_{\pi} \mid \pi \in P\right\}$ of disjoint compact invariant subsets of $S$ such that if $x \in S \backslash \bigcup_{\pi \in P} M_{\pi}$, then there are $\pi, \pi^{\prime} \in P$, $\pi<\pi^{\prime}$ with $x \in C\left(M_{\pi^{\prime}}, M_{\pi}\right)$.

Given a Morse decomposition $\left\{M_{\pi} \mid \pi \in(P,<)\right\}$, there is a minimal partial order on $P,<_{F}$, defined by taking the transitive closure of the relation: $\pi<_{F} \pi^{\prime}$ if $C\left(M_{\pi^{\prime}}, M_{\pi}\right) \neq \varnothing$. We call $<_{F}$ the flow-defined partial order on $P$. 
Let $I \in \mathscr{I}(P,<)$. Define

$$
M(I)=\left(\bigcup_{\pi \in P} M_{\pi}\right) \cup\left(\bigcup_{\pi, \pi^{\prime} \in P} C\left(M_{\pi^{\prime}}, M_{\pi}\right)\right) .
$$

LeMma 2.4 (see [12]). If $I \in \mathscr{I}(P)$, then $M(I)$ is an isolated invariant set. If $I \in \mathscr{A}(P)$, then $M(I)$ is an attractor in $S$ with dual repeller $M(P \backslash I)$. The set $\left\{M_{\pi} \mid \pi \in I\right\}$ is $a$ Morse decomposition of $M(I)$.

COROLlary 2.5. If $(I, J)$ is an adjacent pair, then $(M(I), M(J))$ is an attractorrepeller pair in $M(I J)$.

Suppose $\Lambda$ is an interval in $\mathbf{R}$ and $X \times \Lambda$ is a parametrized family of local flows, i.e. $X \times \Lambda$ is a local flow and $X \times\{\lambda\}$ is a local flow for each $\lambda \in \Lambda$. We have the obvious projections $p_{X}: X \times \Lambda \rightarrow X, p_{\Lambda}: X \times \Lambda \rightarrow \Lambda$.

Suppose that for all $\lambda$ in some compact subinterval $\Lambda^{\prime}$ we have an isolated invariant set $S^{\lambda}$, and for $\lambda \neq \mu, S^{\lambda}$ and $S^{\mu}$ are related by continuation.

Definition 2.6. We say the collection $\left\{M_{\pi}^{\lambda} \mid \pi \in P, \lambda \in \Lambda^{\prime}\right\}$ is a Morse decomposition for the family $\left\{S^{\lambda} \mid \lambda \in \Lambda^{\prime}\right\}$ which continues for all $\lambda$ if:

(a) for each $\lambda \in \Lambda^{\prime},\left\{M_{\pi}^{\lambda} \mid \pi \in P\right\}$ is a Morse decomposition for $S^{\lambda}$;

(b) for each $\mu, \lambda \in \Lambda^{\prime}, \pi \in P$, the sets $M_{\pi}^{\lambda}$ and $M_{\pi}^{\mu}$ are related by continuation. Let $<_{\lambda}$ denote the flow-defined order on $\left\{M_{\pi}^{\lambda} \mid \pi \in P\right\}$. We do not assume that the flow-defined order is the same for all $\lambda$.

\section{Connections in one-parameter families of flows}

Let $\delta>0, \Lambda=(-2 \delta, 1+2 \delta)$. Let $\dot{x}=f(x, \lambda)$ be a one-parameter family of differential equations on $\mathbf{R}^{d}$ parametrized by $\Lambda$. We think of beginning with a system of equations parametrized by $[0,1]$ and extending in some reasonable way. For each $\lambda$ let $\phi_{t}^{\lambda}(x)$ denote the local flow generated by $\dot{x}=f(x, \lambda)$.

Suppose $N \subset \mathbf{R}^{d}$ is a compact isolating neighbourhood for each $\lambda$. Let $S^{\lambda}$ denote the maximal invariant set contained in $N$ in the flow $\phi_{t}^{\lambda}$.

We now consider the perturbed equations

$$
\dot{x}=f(x, \lambda), \quad \dot{\lambda}=g(\lambda),
$$

where $g$ is smooth, $g^{\prime}(0)<0, g^{\prime}(1)>0$, and

$$
g(\lambda) \begin{cases}<0 & 0<\lambda<1, \\ =0 & \lambda=0,1, \\ >0 & \lambda<0 \text { or } \lambda>1 .\end{cases}
$$

For example, $g(\lambda)=\epsilon \lambda(1-\lambda)$. Let $\|g\|$ denote the sup norm of $g$. Let $\Phi_{t}(x, \lambda)$ denote the local flow generated by (3.1).

Proposition 3.2. If $\|g\|$ is sufficiently small, then $N \times[-\delta, 1+\delta]$ is an isolating neighbourhood for $\Phi_{1}$.

Proof. The proof follows from a standard compactness argument, so we omit it.

Assume $\|g\|$ is small and let $\hat{S}$ be the maximal invariant set in $N \times[-\delta, 1+\delta]$. Clearly $\hat{S} \subset N \times[0,1]$. We use Morse decompositions of $S^{0}$ and $S^{1}$ to make a Morse 
decomposition of $\hat{S}$. To establish notation, if $A \subset \mathbf{R}^{d}, \lambda \in \Lambda$, then $A^{\lambda}$ will denote the set $A \times\{\lambda\} \subset \mathbf{R}^{d} \times \Lambda$. The following lemma follows from standard compactness facts, so we omit the proof.

LEMMA 3.3. Let $A \subset \mathbf{R}^{d}$ be an isolated invariant set for the flow $\phi_{t}^{0}$. Then $A^{0}$ is isolated in $\Phi_{t}$.

Of course, $A^{1}$ is also isolated in $\Phi_{t}$.

LEMMA 3.4. Let $\left\{M_{\pi} \mid \pi \in\left(P,<_{P}\right)\right\}$ be a Morse decomposition of $S^{0}$ and $\left\{M_{\rho} \mid \rho \in\left(R,<_{R}\right)\right\}$ be a Morse decomposition of $S^{1}$. Then the collection $\left\{M_{\pi}^{0} \mid \pi \in P\right\} \cup$ $\left\{M_{p}^{1} \mid \rho \in R\right\}$ is a Morse decomposition for $\hat{S}$. An admissible ordering is given by

$$
\begin{array}{ll}
M_{\pi}^{0}<M_{\rho}^{1} & \text { for all } \pi \in P, \rho \in R, \\
M_{\rho}^{1}<M_{\rho^{\prime}}^{1} & \text { if } \rho<{ }_{R} \rho^{\prime}, \\
M_{\pi}^{0}<M_{\pi^{\prime}}^{0} & \text { if } \pi<_{P} \pi^{\prime} .
\end{array}
$$

Proof. $M_{\pi}^{0}$ and $M_{\rho}^{1}$ are isolated invariant sets by lemma 3.3. Let $(x, \lambda) \in \hat{S}$. If $\lambda=0$, then $\omega(x, \lambda) \subset M_{\pi}^{0}$ and $\omega^{*}(x, \lambda) \subset M_{\pi^{\prime}}^{0}$ with $\pi<_{P} \pi^{\prime}$ since $\Phi_{t}(x, 0)=\left(\phi_{t}^{0}(x), 0\right)$. A similar statement holds if $\lambda=1$. The only other possibility is $0<\lambda<1$. Then clearly $\omega(x, \lambda) \subset S^{0}$ and $\omega^{*}(x, \lambda) \subset S^{1}$. We show $\omega(x, \lambda) \subset M_{\pi}^{0}$ for some $\pi \in P . \omega(x, \lambda)$ is a compact, non-empty, connected, invariant subset of $S^{0}$. So $\omega(x, \lambda) \cap M_{\pi}^{0} \neq \varnothing$ for some $\pi \in P$.

Suppose $\omega(x, \lambda) \not \subset M_{\pi}^{0}$. Then there is a $\pi^{\prime} \neq \pi$ with $\omega(x, \lambda) \cap M_{\pi^{\prime}}^{0} \neq \varnothing$. Without loss of generality, assume $\pi^{\prime} \nless_{P} \pi$. Let $I=\left\{p \in P \mid p \leq_{P} \pi\right\}$. Then $I$ is an attracting interval and $\pi^{\prime} \notin I$. Let $U \subset \mathbf{R}^{d}$ be an isolating neighbourhood for $M(I)$ and let $\hat{U}=U \times \Lambda$. Then $\hat{U} \cap M_{\pi^{\prime}}^{0}=\varnothing$. Choose a sequence $t_{n} \rightarrow \infty$ such that $\Phi_{t_{n}}(x, \lambda) \in \hat{U}$, $\Phi_{t_{n}}(x, \lambda) \rightarrow(y, 0) \in M_{\pi}^{0}$ but $\Phi_{t}(x, \lambda) \notin \operatorname{int}(\hat{U})$ for some $t \in\left(t_{n}, t_{n+1}\right)$. We can choose such a sequence because $\omega(x, \lambda) \cap M_{\pi^{\prime}}^{0} \neq \varnothing$. Now choose a sequence $s_{n} \in\left(t_{n}, t_{n+1}\right)$ such that

$$
\Phi_{s_{n}}(x, \lambda) \in \partial \hat{U} \quad \text { and } \quad \Phi_{t}(x, \lambda) \in \operatorname{cl}(\hat{U}) \quad \text { for all } t \in\left[t_{n}, s_{n}\right] .
$$

Let $(z, \mu)$ be a limit point of $\left\{\Phi_{s_{n}}(x, \lambda)\right\}$. Since $s_{n} \rightarrow \infty, \mu=0$ and $(z, 0) \in(\partial U)^{0}$. The sequence $s_{n}-t_{n}$ is unbounded, otherwise $(z, 0)$ lies on the orbit of $(y, 0)$ and this orbit is contained in the interior of $\hat{U}$. It follows that $\Phi_{r}(z, 0) \in \operatorname{cl}\left(U^{0}\right)$ for all $t \leq 0 .(z, 0) \in S^{0}$ since $(x, \lambda) \in \hat{S}$, so $\omega(z, 0) \subset M_{\pi^{\prime \prime}}^{0}$ for some $\pi^{\prime \prime} \in P$. If $\pi^{\prime \prime} \in I$, then this contradicts the fact that $U$ is an isolating neighbourhood. If $\pi^{\prime \prime} \notin I$, then this contradicts the fact that $M(I)$ is an attractor. In either case we have a contradiction and we conclude that $\omega(x, \lambda)$ is contained in $M_{\pi}^{0}$ for some $\pi \in P$. A similar argument establishes that $\omega^{*}(x, \lambda)$ is contained in $M_{p}^{1}$ for some $\rho \in R$.

Suppose that we have a connection from $M_{\rho}^{1}$ to $M_{\pi}^{0}$ in $\Phi_{t}$, and that a connection persists as we let $\|g\| \rightarrow 0$ in equation (3.1). One could hope that the $M_{\rho}^{1}$ to $M_{\pi}^{0}$ connections would give information about connections for $\lambda \in(0,1)$. For example, if $\boldsymbol{M}_{\pi}^{0}$ continues to $\boldsymbol{M}_{\pi}^{\lambda}$ and $\boldsymbol{M}_{\rho}^{1}$ continues to $\boldsymbol{M}_{\rho}^{\lambda}$ for all $\lambda$, does it follow that $\boldsymbol{M}_{\rho}^{\lambda}$ and $M_{\pi}^{\lambda}$ are connected for some value of $\lambda$ ? This is not true in general, but one can draw some conclusion about connections for $\lambda \in(0,1)$ as in theorem 3.13 below. In order to use the information, we construct a limiting 'connection' as $\|g\| \rightarrow 0$. 
Let $g_{n}$ be a sequence of functions satisfying the conditions on $g$ in (3.1) and assume that $\|g\| \rightarrow 0$. Consider the sequence of equations

$$
\dot{x}=f(x, \lambda), \quad \dot{\lambda}=g_{n}(\lambda)
$$

and let $\Phi_{t}^{n}$ be the local flow generated by equation (3.1n). The proof of the following lemma is straightforward so we omit it.

LEMMA 3.5. Let $\Phi_{t}^{\infty}$ denote the flow generated by (3.1) with $g \equiv 0$. If $x_{n}$ and $t_{n}$ are bounded sequences, $x_{n} \rightarrow x, t_{n} \rightarrow t$ and $\lambda_{n} \rightarrow \lambda$, then $\Phi_{t_{n}}^{n}\left(x_{n}, \lambda_{n}\right) \rightarrow \Phi_{1}^{\infty}(x, \lambda)$.

Assume now that for each $n$ there is a connection $c_{n}$ from $M_{\rho}^{1}$ to $M_{\pi}^{0}$ in the local flow $\Phi_{1}^{\infty}$. Let

$$
\bar{c}_{n}=\text { closure of } c_{n}=c_{n} \cup \omega\left(c_{n}\right) \cup \omega^{*}\left(c_{n}\right) .
$$

$\bar{c}_{n}$ is compact, invariant and connected. In the Hausdorff topology on $N \times[0,1], \bar{c}_{n}$ has a convergent subsequence which we again label $\bar{c}_{n}$. Let $\bar{c}$ denote the limit of $\bar{c}_{n}$.

LемMа 3.6. $\bar{c}$ is compact, connected and invariant in $\Phi_{1}^{\infty}$.

Proof. Compactness is immediate; connectedness follows from lemma 1.2. It remains to prove invariance.

Let $\hat{d}$ denote the usual metric on $\mathbf{R}^{d} \times \Lambda \subset \mathbf{R}^{d+1}$ and let $\rho$ denote the Hausdorff metric on the compact subsets of $N \times \Lambda$. Suppose $\bar{c}$ is not invariant. Then there is a point $(x, \lambda) \in \bar{c}$ and a $t \in \mathbf{R}$ with $\hat{d}\left(\Phi_{1}^{\infty}(x, \lambda), \bar{c}\right)=\varepsilon>0$. By choosing $n$ large, we can find $\eta>0$ such that if $\hat{d}((x, \lambda),(y, \mu))<\eta$, then $\hat{d}\left(\left(\Phi_{t}^{\infty}(x, \lambda), \Phi_{t}^{\infty}(y, \mu)\right)<\varepsilon / 3\right.$ and for any $(y, \mu) \in N \times \Lambda, \hat{d}\left(\Phi_{t}^{\infty}(y, \mu), \Phi_{t}^{n}(y, \mu)\right)<\varepsilon / 3$. Also, if $n$ is sufficiently large, $\rho\left(\bar{c}_{n}, \bar{c}\right)<\min \{\varepsilon / 3, \eta\}$. So choose $n$ large and let $(y, \mu) \in \bar{c}_{n}$ with $\hat{d}((x, \lambda),(y, \mu))<\eta$. Then

$$
\begin{aligned}
\hat{d}\left(\Phi_{t}^{\infty}(x, \lambda), \bar{c}\right) \leq & \hat{d}\left(\Phi_{t}^{\infty}(x, \lambda), \Phi_{t}^{\infty}(y, \mu)\right)+\hat{d}\left(\Phi_{t}^{\infty}(y, \mu), \Phi_{t}^{n}(y, \mu)\right) \\
& +\hat{d}\left(\Phi_{t}^{n}(y, \mu), \bar{c}_{n}\right)+\rho\left(\bar{c}_{n}, \bar{c}\right) \\
& <\frac{\varepsilon}{3}+\frac{\varepsilon}{3}+0+\frac{\varepsilon}{3}=\varepsilon,
\end{aligned}
$$

so we have a contradiction and we conclude $\bar{c}$ is invariant.

Let $\bar{c}^{\lambda}=\bar{c} \cap\left(\mathbf{R}^{d} \times\{\lambda\}\right)$. Then $\bar{c}^{\lambda} \neq \varnothing, 0 \leq \lambda \leq 1, \bar{c}^{0} \subset M_{\pi}^{0}$ and $\bar{c}^{1} \subset M_{\rho}^{1}$.

LеммА 3.7. $\bar{c}^{\lambda}$ is non-empty, compact, connected and invariant in $\Phi_{t}^{\infty}$.

Proof. $\bar{c}^{\lambda} \neq \varnothing$ because $\bar{c}$ is connected; $\bar{c}^{\lambda}$ is compact and invariant because it is the intersection of the compact invariant set $\bar{c}$ and the closed invariant set $\boldsymbol{R}^{d} \times\{\lambda\}$. The proof of connectedness is straightforward, so we omit it.

Suppose for each $\lambda \in[0,1]$ we have a Morse decomposition $\left\{M_{\pi}^{\lambda} \mid \pi \in\left(P_{\lambda},<_{\lambda}\right)\right\}$ of $S^{\lambda} . \bar{c}^{\lambda}$ is a compact, invariant subset of $N^{\lambda}$, so $\bar{c}^{\lambda} \subset S^{\lambda}$. If $(x, \lambda) \in \bar{c}^{\lambda}$, then so are $\omega(x, \lambda)$ and $\omega^{*}(x, \lambda)$ (limits in the flow $\left.\Phi_{t}^{\infty}\right)$. So $\bar{c}^{\lambda}$ consists of orbits in at least one of the Morse sets $M_{\pi}^{\lambda}$, plus connecting orbits between some of the Morse sets if $\bar{c}^{\lambda}$ intersects more than one Morse set. Let $I^{\lambda}=\left\{\pi \in P_{\lambda} \mid \bar{c}^{\lambda} \cap M_{\pi}^{\lambda} \neq \varnothing\right\}$. Then $I^{\lambda}$ is non-empty since $\bar{c}^{\lambda}$ is compact and invariant. In fact, $I^{\lambda}$ is a totally ordered subset of $\left(P,<_{\lambda}\right)$. 
LEMMA 3.8. Suppose we have a sequence $\left(x_{n}, \lambda_{n}\right) \in \bar{c}_{n},\left(x_{n}, \lambda_{n}\right) \rightarrow(x, \lambda) \in M_{p}^{\lambda}$, and we have a sequence $t_{n} \geq 0$ such that the sequence $\Phi_{t_{n}}\left(x_{n}, \lambda_{n}\right)=\left(y_{n}, \mu_{n}\right)$ converges to $(y, \lambda) \in M_{p^{\prime}}^{\lambda}$. Then in the flow-defined partial order on $P_{\lambda}, p^{\prime} \leq p$.

Proof. If $\left\{t_{n}\right\}$ is bounded, then let $t$ be a limit point of $\left\{t_{n}\right\}$. It follows that $\Phi_{1}^{\infty}(x, \lambda)=$ $(y, \lambda)$, so $p^{\prime}=p$ in this case.

Assume now that $t_{n} \rightarrow \infty$, and suppose $p^{\prime} \not p$ in the flow-defined order on $P_{\lambda}$. We will derive a contradiction. Let

$$
I=\left\{q \in P_{\lambda} \mid q \leq p \text { in the flow-defined order on } P_{\lambda}\right\} .
$$

Then $I$ is an attracting interval in $P_{\lambda}$ with the flow-defined order, so $M(I)$ is an attractor in the flow $\phi_{1}^{\lambda}$. Let $U \subset \mathbf{R}^{d}$ isolate $M(I)$ and let $\hat{U}=U \times \Lambda$. For $n$ sufficiently large, $\left(x_{n}, \lambda_{n}\right) \in \hat{U}$ but $\left(y_{n}, \mu_{n}\right) \notin \mathrm{cl}(\hat{U})$. So we can find $s_{n} \in\left(0, t_{n}\right)$ such that

$$
\Phi_{s_{n}}^{n}\left(x_{n}, \lambda_{n}\right)=\left(z_{n}, \nu_{n}\right) \in \partial \hat{U} \quad \text { and } \quad \Phi_{t}^{n}\left(x_{n}, \lambda_{n}\right) \in \hat{U} \quad \text { for } t \in\left[0, s_{n}\right] .
$$

Let $(z, \nu)$ be a limit point of $\left\{\left(z_{n}, \nu_{n}\right)\right\}$. Since $\lambda_{n} \leq \nu_{n} \leq \mu_{n}, \nu=\lambda$. The sequence $\left\{s_{n}\right\}$ is unbounded: if not, choose a limit point $s$ and we have $\Phi_{s}^{\infty}(x, \lambda) \in \partial \hat{U}$ which is impossible.

We claim that for any $t>0, \Phi_{-1}^{\infty}(z, \lambda) \in \operatorname{cl}\left(U^{\lambda}\right)$. If not, then $\Phi_{-\tau}^{\infty}(z, \lambda) \notin \operatorname{cl}\left(U^{\lambda}\right)$ for some $\tau>0$. It follows that for all $n$ sufficiently large, $\Phi_{-\tau}^{n}\left(z_{n}, \nu_{n}\right) \notin \operatorname{cl}(\hat{U})$. But $\Phi_{-\tau}^{n}\left(z_{n}, \nu_{n}\right)=\Phi_{s_{n}-\tau}^{n}\left(x_{n}, \lambda_{n}\right) \in \hat{U}$ if $s_{n}>\tau$, which is the case for $n$ sufficiently large. Thus $\Phi_{-,}^{\infty}(z, \nu) \in \mathrm{cl}\left(U^{\lambda}\right)$ for all $t \geq 0$.

So $\omega^{*}(z, \nu) \subset M(I) \times\{\lambda\}$ (limit in the $\Phi_{t}^{\infty}$ flow). $\Phi_{t}^{\infty}(z, \nu) \in N^{\lambda}$ for all $t \leq 0$ since $(z, \nu)$ is the limit of points on the $\bar{c}_{n}$ so $\omega(z, \nu) \in M_{q}^{\lambda}$ for some $q \in P_{\lambda}$. If $q \in I$, then this contradicts the fact that $U$ is an isolating neighbourhood for $\phi^{\lambda}$. If $q \notin I$, then we contradict the fact that $M(I)$ is an attractor. In either case we have a contradiction, so the lemma is proved.

An analogous argument holds for the backward flow, i.e. $t_{n} \leq 0$. We then obtain $p \leq p^{\prime}$ in the conclusion of lemma 3.8 .

LEMMA 3.9. For each $\lambda \in[0,1], I^{\lambda}$ is a totally ordered subset of $P_{\lambda}$ in the flow-defined order on $P_{\lambda}$.

Proof. Suppose $p, p^{\prime} \in I^{\lambda}$. Let $(x, \lambda) \in M_{p}^{\lambda},(y, \lambda) \in M_{p^{\prime}}^{\lambda}$. Then there are sequences $\left(x_{n}, \lambda_{n}\right) \in \bar{c}_{n},\left(y_{n}, \mu_{n}\right) \in \bar{c}_{n},\left(x_{n}, \lambda_{n}\right) \rightarrow(x, \lambda),\left(y_{n}, \mu_{n}\right) \rightarrow(y, \lambda)$. There exist times $t_{n}$ with $\Phi_{t_{n}}^{n}\left(x_{n}, \lambda_{n}\right)=\left(y_{n}, \mu_{n}\right)$. By taking a subsequence, we may assume that all the $t_{n}$ are non-negative or all the $t_{n}$ are non-positive. If all the $t_{n}$ are non-negative, lemma 3.8 implies $p^{\prime} \leq p$, and if the $t_{n}$ are non-positive we have $p \leq p^{\prime}$. In any case, $p$ and $p^{\prime}$ are comparable in the flow-defined order.

Now assume that the Morse decomposition $\left\{M_{\pi}^{\lambda} \mid \pi \in\left(P,<_{\lambda}\right)\right\}$ continues over the entire interval $\Lambda$.

LEMMA 3.10 (see [12]). For each $\pi \in P, \bigcup_{\lambda \in[0,1]} M_{\pi}^{\lambda}$ is a compact isolated invariant set on the flow $\Phi_{t}^{\infty}$ on $\mathbf{R}^{n} \times[0,1]$.

For $\pi \in P$, define the sets

$$
A_{\pi}=\left\{\lambda \in[0,1] \mid \bar{c}^{\lambda} \subset M_{\pi}^{\lambda}\right\}, \quad B_{\pi}=\left\{\lambda \in[0,1] \mid \bar{c}^{\lambda} \cap M_{\pi}^{\lambda} \neq \varnothing\right\} .
$$


Clearly $A_{\pi} \subset B_{\pi}$. If we have a connection from $M_{\rho}^{1}$ to $M_{\pi}^{0}$ for all $n$ in (3.1n), then $0 \in A_{\pi}$ and $1 \in A_{\rho}$.

LeMma 3.11. For each $\pi \in P, A_{\pi}$ is open in $[0,1]$ and $B_{\pi}$ is compact.

Proof. $B_{\pi}=p_{\Lambda}\left(\bar{c} \cap \bigcup_{\lambda \in \Lambda} M_{\pi}^{\lambda}\right)$ and $\bar{c} \cap\left(\bigcup_{\lambda \in \Lambda} M_{\pi}^{\lambda}\right)$ is compact. $A_{\pi}=\Lambda \backslash\left(\bigcup_{\pi^{\prime} \neq \pi} B_{\pi^{\prime}}\right)$ because $\bar{c}^{\lambda}$ is non-empty, compact and invariant.

The following proposition completes the description of $\bar{c}$.

Proposition 3.12. Let $\lambda \in(0,1)$. Let $p=\inf I^{\lambda}, p^{\prime}=\sup I^{\lambda}$. Then there is an $\varepsilon>0$ such that $(\lambda-\varepsilon, \lambda) \subset A_{p}$ and $(\lambda, \lambda+\varepsilon) \subset A_{p}$.

Proof. We prove the statement for $A_{p}$. The proof for $A_{p^{\prime}}$ is the same using the backward flow. Suppose that the proposition is false. Then there is a sequence of points $\lambda_{m}$ increasing, $\lambda_{m} \rightarrow \lambda$ with $\lambda_{m} \in B_{r_{m}}$ for some $r_{m} \in P \backslash\{p\}$. Since $P$ is finite, we can choose a subsequence which we again label $\lambda_{m}$ such that $\lambda_{m} \in B_{q}$ for some $q \in P \backslash\{p\}$. By compactness, $\lambda \in B_{q}$. Choose sequences $\left(x_{n}, \mu_{n}\right) \in \bar{c}_{n}$ and $\left(y_{n}^{m}, \nu_{n}^{m}\right) \in \bar{c}_{n}$ for each $m$ such that $\left(x_{n}, \mu_{n}\right) \rightarrow(x, \lambda) \in M_{p}^{\lambda}$ and $\left(y_{n}^{m}, \nu_{n}^{m}\right) \rightarrow\left(y, \lambda_{n}\right)$ in $M_{q}^{\lambda_{m}}$. Choose a subsequence $\left(x_{n_{m}}, \mu_{n_{m}}\right)$ such that
(a) $n_{m+1}>n_{m}$,
(b) $d\left(\left(y_{n_{m}}^{m}, \nu_{n_{m}}^{m}\right), M_{q}^{\lambda_{m}}\right)<1 / m$,
(c) $\mu_{n_{m}}<\nu_{n_{m}}^{m}$.

We can choose such a subsequence because $\lambda_{m}<\lambda$. Choose a subsequence of $\left(y_{n_{m}}^{m}, \nu_{n_{m}}^{m}\right) \rightarrow(y, \nu)$. We have $\nu=\lambda$ since $\lambda_{m} \rightarrow \lambda$. Also $(y, \lambda) \in M_{q}^{\lambda}$ since $\bigcup_{\lambda \in[0,1]} M_{q}^{\lambda}$ is compact. Since $\mu_{n_{m}}<\nu_{n_{m}}$, there are times $t_{m} \geq 0$ such that $\Phi_{r_{m}}^{n_{m}}\left(x_{n_{m}}, \mu_{n_{m}}\right)=$ $\left(y_{n_{m}}^{m}, \nu_{n_{m}}^{m}\right)$. By lemma 3.8, $q \leq_{\lambda} p$ and, since $q \neq p$, we have $q<_{\lambda} p . q \in I^{\lambda}$ by lemma 1.1. But this contradicts the fact that $p=\inf I^{\lambda}$.

We can now use $\bar{c}$ to get information about connections for parameter values $\lambda \in(0,1)$.

THEOREM 3.13. Suppose that for all $n$ there is a connection from $M_{\rho}^{1}$ to $M_{\pi}^{0}$. Then there exist finite sequences $1>\lambda_{1}>\lambda_{2}>\cdots>\lambda_{k}>0$ and $\rho=\pi_{1}, \pi_{2}, \ldots, \pi_{k+1}=\pi$ such that $M_{\pi_{i+1}}^{\lambda_{i}}<_{i} M_{\pi_{i}}^{\lambda_{i}}, i=1, \ldots, k$, where $<_{i}$ is the flow-defined order on $P$ for the flow $\phi_{i}^{\lambda_{i}}$.

Proof. Take a convergent subsequence $\bar{c}_{n} \rightarrow \bar{c}$ in the Hausdorff metric. By lemma 3.11 and proposition 3.12 , the set $[0,1] \backslash \bigcup_{p \in P} A_{p}$ is discrete and compact, hence finite. These are the $\lambda_{i} .0 \in A_{\pi}$ and $1 \in A_{\rho}$ so each $\lambda_{i}$ is in $(0,1)$. Let $\pi_{i}=\sup I^{\lambda_{i}}=$ $\inf I^{\lambda_{i-1}}$ since $\left(\lambda_{i+1}, \lambda_{i}\right) \subset A_{\pi_{i+1}}$ for each $i . \pi_{1}=\rho$ and $\pi_{k+1}=\pi$ since $0 \in A_{\pi}, 1 \in A_{\rho}$ and $A_{\pi}$ and $A_{\rho}$ are open.

This result is the best possible. There are examples where $M_{\rho}^{1}$ is connected to $M_{\pi}^{0}$ for all $n$ but $\pi \not_{\lambda} \rho$ for each $\lambda \in[0,1]$. These examples will be discussed in a future paper.

\section{The connection matrix}

In this section we discuss the connection matrix, an algebraic object which contains information about connecting orbits between the sets in a Morse decomposition. Most of the results of this section are due to Franzosa.

Let $\Gamma$ be a Hausdorff space with a flow $(x, t) \mapsto x \cdot t$. For $J \subset \mathbf{R}, x \cdot J=\{x \cdot t \mid t \in J\}$. Let $X \subset \Gamma$ be a local flow. 
Definition 4.1. Let $Z \subset Y \subset X$. We say $Z$ is a positively invariant relative to $Y$ if $x \in Z$ and $x \cdot[0, t] \subset Y$ implies $x \cdot[0, t] \subset Z$.

Let $S \subset X$ be an isolated invariant set.

Definition 4.2. A compact pair $\left(N_{1}, N_{0}\right) \subset X$ is an index pair for $S$ if:

(i) $N_{1} \backslash N_{0}$ is a neighbourhood of $S$ and cl $\left(N_{1} \backslash N_{0}\right)$ is an isolating neighbourhood for $S$;

(ii) $N_{0}$ is positively invariant relative to $N_{1}$;

(iii) if $x \in N_{1}$ and $x \cdot[0, \infty] \not \subset N_{1}$, then there is a $t>0$ with $x \cdot[0, t] \subset N_{1}$ and $x \cdot t \in N_{0}$.

Theorem 4.3 (see [1]). Index pairs exist, and if $\left(N_{1}, N_{0}\right)$ and $\left(N_{1}^{\prime}, N_{0}^{\prime}\right)$ are two index pairs for $S$, then there is a flow-defined homotopy equivalence between the pointed quotient spaces $N_{1} / N_{0}$ and $N_{1}^{\prime} / N_{0}^{\prime}$.

The homotopy type $\left[N_{1} / N_{0}\right]$ is called the Conley index of $S$ and is denoted $h(S)$. References for the Conley index include [1], [2], [11] and [12].

Let $\left(A, A^{*}\right)$ be an attractor-repeller pair in $S$ and let $\left(N_{2}, N_{1}, N_{0}\right)$ be a compact triple in $X$.

Proposition 4.4 (see [5]). If $\left(N_{2}, N_{0}\right)$ is an index pair for $S$ and $\left(N_{1}, N_{0}\right)$ is an index pair for $A$, then $\left(N_{2}, N_{1}\right)$ is an index pair for $A^{*}$.

We call $\left(N_{2}, N_{1}, N_{0}\right)$ an index triple. The inclusion and projection maps (of pointed spaces)

$$
N_{1} / N_{0} \stackrel{i}{\rightarrow} N_{2} / N_{0} \stackrel{p}{\rightarrow} N_{2} / N_{1}
$$

induce maps on the singular chains

$$
C\left(N_{1} / N_{0}\right) \stackrel{i}{\rightarrow} C\left(N_{2} / N_{0}\right) \stackrel{p}{\rightarrow} C\left(N_{2} / N_{1}\right)
$$

which induce a long exact homology sequence

$$
\cdots \rightarrow H_{q}\left(N_{1} / N_{0}\right) \stackrel{i}{\rightarrow} H_{q}\left(N_{2} / N_{0}\right) \stackrel{p}{\rightarrow} H_{q}\left(N_{2} / N_{1}\right) \stackrel{\partial}{\rightarrow} H_{q-1}\left(N_{1} / N_{0}\right) \rightarrow \cdots
$$

The homology groups are relative to a base point. We consider coefficients in a field F. The boundary map $\partial: H_{q}\left(N_{2} / N_{1}\right) \rightarrow H_{q-1}\left(N_{1} / N_{0}\right)$ contains connection information.

Proposition 4.6 (see [4]). If $\partial: H_{q}\left(N_{2} / N_{1}\right) \rightarrow H_{q-1}\left(N_{1} / N_{0}\right)$ is not identically 0 , then $C\left(A^{*}, A\right) \neq \varnothing$.

In [7] Kurland showed that sequence (4.5) is natural in the sense that if $\left(N_{2}^{\prime}, N_{1}^{\prime}, N_{0}^{\prime}\right)$ is another index triple, then there are isomorphisms $H_{q}\left(N_{1} / N_{0}\right) \rightarrow$ $H_{q}\left(N_{1}^{\prime} / N_{0}^{\prime}\right)$, etc., which come from flow-defined homotopy equivalences on the quotient spaces, such that the following diagram commutes:

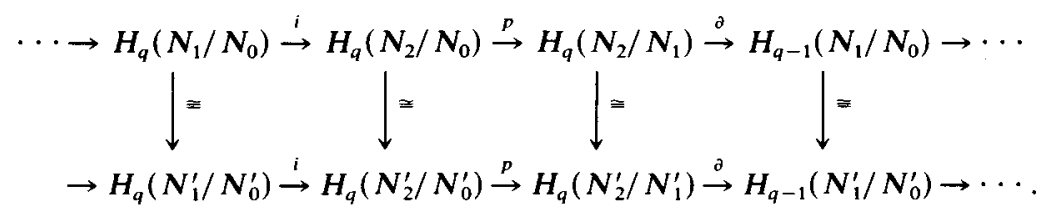


In view of this naturality, we rewrite sequence (4.5) as

$$
\cdots \rightarrow H_{q}(A) \stackrel{i}{\rightarrow} H_{q}(S) \stackrel{p}{\rightarrow} H_{q}\left(A^{*}\right) \stackrel{\partial}{\rightarrow} H_{q-1}(A) \rightarrow \cdots,
$$

where it is implicit that we have chosen an index triple, and the sequence for any other triple is related by (4.7).

Now suppose that $\left\{M_{\pi} \mid \pi \in(P,<)\right\}$ is a Morse decomposition of $S$. If $(I, J)$ is an adjacent pair of intervals in $P$, then $(M(I), M(J))$ is an attractor-repeller pair in $M(I J)$ so we have a sequence as in (4.8). We want to construct an analogue of an index triple which works for all of these attractor-repeller pairs.

Definition 4.9. An index filtration for the Morse decomposition $\left\{M_{\pi} \mid \pi \in(P,<)\right\}$ is a collection $\{N(I) \mid I \in \mathscr{A}(P)\}$ of compact subsets of $X$ indexed by the attracting intervals of $P$ such that:

(a) for all $I \in \mathscr{A}(P),(N(I), N(\varnothing))$ is an index pair for $M(I)$;

(b) $N\left(I_{1} \cap I_{2}\right)=N\left(I_{1}\right) \cap N\left(I_{2}\right)$ for all $I_{1}, I_{2} \in \mathscr{A}(P)$;

(c) $N\left(I_{1} \cup I_{2}\right)=N\left(I_{1}\right) \cup N\left(I_{2}\right)$ for all $I_{1}, I_{2} \in \mathscr{A}(P)$.

An index filtration actually gives us index pairs for all $M(I), I \in \mathscr{I}(P)$ by the following two propositions.

Proposition 4.10. Suppose $J \in \mathscr{I}(P)$. Then there is an interval $I \in \mathscr{A}(P)$ with $(I, J)$ an adjacent pair of intervals and $I \cup J \in \mathscr{A}(P)$.

Proposition 4.11. Suppose $J \in \mathscr{I}(P), I$ as in proposition 4.10. Then $(N(I J), N(I))$ is an index pair for $M(J)$. If $I_{1}$ and $I_{2}$ are two such intervals, then the pointed spaces $N\left(I_{1} J\right) / N\left(I_{1}\right)$ and $N\left(I_{2} J\right) /\left(N\left(I_{2}\right)\right.$ are homeomorphic.

The proofs of propositions 4.10 and 4.11 are straightforward, or see [4]. The existence of index filtrations was shown by Franzosa in [5].

For any $J \in \mathscr{I}(P)$, we define the chain complex $C(I)=C(N(I J) / N(J))$ for any $I$ satisfying definition 4.9 . For any adjacent pair of intervals $(I, J)$, there are inclusion and projection maps

$$
C(I) \stackrel{i}{\rightarrow} C(J) \stackrel{p}{\rightarrow} C(J)
$$

which induce a long exact homology sequence

$$
\cdots H_{q}(I) \stackrel{i}{\rightarrow} H_{q}(I J) \stackrel{p}{\rightarrow} H_{q}(J) \stackrel{\partial}{\rightarrow} H_{q-1}(I) \rightarrow \cdots
$$

and $\partial$ contains connection information.

Definition 4.13. Let $(P,<)$ be a partially ordered set. A collection

$\{H(I), i(I, I J), p(I J, I), \partial(J, I) \mid I \in \operatorname{cl}(P)$ and $(I, J)$ an adjacent pair of intervals $\}$

of graded vector spaces $H(I)$ and maps $i: H(I) \rightarrow H(I J), p: H(I J) \rightarrow H(J)$ of degree 0 and $\partial: H(J) \rightarrow H(I)$ of degree -1 is a homology braid if the maps are such that for each adjacent pair of intervals $(I, J)$ the sequence $(4.12)$ is exact. We denote the collection by $\mathscr{H}$. We call $\mathscr{H}$ flow-generated if the $H(I)$ and maps come from an index filtration as above.

Our goal is to condense the information in the sequence (4.12) into maps between the homologies of the indices of the single $M_{\pi}$. 
For $I \in \mathscr{I}(P)$, let $C \Delta(I)=\bigoplus_{\pi \in I} H(\pi)$, with the obvious grading $(C \Delta(I))_{q}=$ $\oplus_{\pi \in I} H_{q}(\pi)$.

Definition 4.14. $\Delta: C \Delta(P) \rightarrow C \Delta(P)$ a homomorphism is called an upper triangular boundary map if:

(a) $\Delta^{2}=0$;

(b) $\Delta$ has degree -1 ;

(c) the restriction $\Delta\left(\pi^{\prime}, \pi\right): H\left(\pi^{\prime}\right) \rightarrow H(\pi)$ is 0 if $\pi \nless \pi^{\prime}$.

Let $\Delta(I)$ denote the restriction of $\Delta$ to $C \Delta(I)$. It is not difficult to see that if $\Delta$ is an upper triangular boundary map, then so is $\Delta(I)$. Let $H \Delta(I)$ denote the homology of the chain complex $(C \Delta(I), \Delta(I))$. If $(I, J)$ is an adjacent pair of intervals, then we have an obvious inclusion and projection

$$
C \Delta(I) \stackrel{i}{\rightarrow} C \Delta(I J) \stackrel{p}{\rightarrow} C \Delta(J)
$$

which give us a long exact homology sequence

$$
\cdots \rightarrow H_{q} \Delta(I) \stackrel{i}{\rightarrow} H_{q} \Delta(I J) \stackrel{p}{\rightarrow} H_{q} \Delta(J) \stackrel{\bar{\Delta}}{\rightarrow} H_{q-1} \Delta(I) \rightarrow \cdots .
$$

It is not hard to see that $\bar{\Delta}$ is the map induced by $\Delta$. We can now define the connection matrix.

Definition 4.16. Let $\mathscr{H}$ be a homology braid. Then an upper triangular boundary map $\Delta$ is called a connection matrix for $\mathscr{H}$ if, for each $I \in \mathscr{I}(P)$, there is an isomorphism $\Phi(I): H \Delta(I) \rightarrow H(I)$ satisfying:

(a) for $\pi \in P, \Phi(\pi): H \Delta(\pi)=H(\pi) \rightarrow H(\pi)$ is the identity;

(b) for all adjacent pairs $(I, J)$, the following commutes:

$$
\begin{aligned}
& \cdots \rightarrow H \Delta(I) \stackrel{i}{\rightarrow} H \Delta(I J) \stackrel{p}{\rightarrow} H \Delta(J) \stackrel{\bar{\Delta}}{\rightarrow} H \Delta(I) \rightarrow \cdots \\
& \downarrow \Phi(I) \quad \downarrow \Phi(I J) \quad \downarrow \Phi(J) \quad \downarrow \Phi(I) \\
& \rightarrow H(I) \stackrel{i}{\rightarrow} H(I J) \stackrel{p}{\rightarrow} H(J) \stackrel{\partial}{\rightarrow} H(I) \rightarrow \cdots,
\end{aligned}
$$

where the top row is (4.15) and the bottom row is (4.12).

Note that if $\pi$ and $\pi^{\prime}$ are adjacent in $P$, i.e. $\left\{\pi, \pi^{\prime}\right\}$ is an interval, then condition (a) says that the connection matrix entry $\Delta\left(\pi^{\prime}, \pi\right)$ is equal to the flow-defined map $\partial: H\left(\pi^{\prime}\right) \rightarrow H(\pi)$. Thus the connection matrix places restrictions on the flow-defined boundary maps. The existence of connection matrices was shown by Franzosa.

THEOREM 4.17 (see [4] and [6]). Given a flow-generated homology braid $\mathscr{H}$, then the set of connection matrices for $\mathscr{H}$ is non-empty.

The connection matrix may not be unique. If the Morse decomposition consists of hyperbolic rest points, then uniqueness of the connection matrix is implied by the transversality condition (see $[\mathbf{1 0}]$ ).

If $I \in \mathscr{I}(P)$ and $\mathscr{H}$ is a homology braid, then there is an obvious restriction of $\mathscr{H}$ to $I$. 
Remark 4.18. If $\Delta$ is a connection matrix for $I$, then $\Delta(I)$ is a connection matrix for the restriction of $H$ to $I$.

Of course, it is not necessarily the case that every connection matrix for $\mathscr{H}$ restricted to $I$ arises in this way.

Definition 4.19. Let $\mathscr{H}$ and $\mathscr{H}^{\prime}=\left\{H^{\prime}(I), i^{\prime}, p^{\prime}, \partial^{\prime} \mid I \in \mathscr{I}(P)\right\}$ be homology braids. We say $\mathscr{H}$ and $\mathscr{H}^{\prime}$ are isomorphic if there are isomorphisms $\theta(I): H(I) \rightarrow H^{\prime}(I)$ for all $I \in \mathscr{I}(P)$ such that the following diagram commutes for all adjacent pairs $(I, J)$ :

$$
\begin{aligned}
& \cdots \rightarrow H(I) \stackrel{i}{\rightarrow} H(I J) \stackrel{p}{\rightarrow} H(J) \stackrel{\partial}{\rightarrow} H(I) \rightarrow \cdots \\
& \downarrow \theta(I) \quad \downarrow \theta(I J) \quad \downarrow \theta(J) \quad \downarrow \theta(I) \\
& \rightarrow H^{\prime}(I) \stackrel{i^{\prime}}{\rightarrow} H^{\prime}(I J) \stackrel{p^{\prime}}{\rightarrow} H^{\prime}(J) \stackrel{\partial^{\prime}}{\rightarrow} H^{\prime}(I) \rightarrow \cdots
\end{aligned}
$$

For example, if $\{N(A) \mid A \in \mathscr{A}(P)\}$ and $\left\{N^{\prime}(A) \mid A \in \mathscr{A}(P)\right\}$ are two index filtrations, then (4.7) implies that the braids $\mathscr{H}$ and $\mathscr{H}^{\prime}$ obtained from the filtrations are isomorphic. If $\mathscr{H}$ and $\mathscr{H}^{\prime}$ are isomorphic, then there is a one-to-one correspondence between their connection matrices.

THEOREM 4.20 (see [4]). If $\mathscr{H}$ and $\mathscr{H}^{\prime}$ are isomorphic braids and $\Delta$ is a connection matrix for $\mathscr{H}$, then $\Delta^{\prime}$ is a connection matrix for $\mathscr{H}^{\prime}$, where

$$
\Delta^{\prime}\left(\pi^{\prime}, \pi\right)=\theta(\pi) \Delta\left(\pi^{\prime}, \pi\right) \theta^{-1}(\pi) \quad \text { for all } \pi, \pi^{\prime} \in P .
$$

We call $\Delta^{\prime}$ the $\theta$-conjugate of $\Delta$. Theorem 4.20 and (4.7) imply that the connection matrix is independent of index filtration, up to conjugation.

Suppose we have a one-parameter family of local flows indexed by $\Lambda$ as in $\S 3$. Suppose that for $\lambda, \mu \in \Lambda$ we have Morse decompositions $\left\{M_{\pi}^{\lambda} \mid \pi \in P\right\}$ and $\left\{M_{\pi}^{\mu} \mid \pi \in P\right\}$ which are related by continuation.

THEOREM 4.21 (see [4]). If the flow-defined partial order on $P$ remains constant as $\left\{M_{\pi}^{\lambda} \mid \pi \in P\right\}$ is continued to $\left\{M_{\pi}^{\mu} \mid \pi \in P\right\}$, then the braids $\mathscr{H}^{\lambda}$ and $\mathscr{H}^{\mu}$ obtained by choosing index filtrations are isomorphic, and each map $\theta(I)$ has degree 0.

It follows that each connection matrix for $\mathscr{H}^{\mu}$ is the conjugate of a connection matrix for $\mathscr{H}^{\lambda}$ by a conjugation of degree 0 .

\section{The transition matrix}

In this section we discuss the theory of the connection matrix for one-parameter families of flows. We will then have an algebraic method of detecting connections from $M_{\rho}^{1}$ to $M_{\pi}^{0}$ in the flow $\Phi_{t}^{n}$.

If we have two topological spaces with flows, then there is an obvious flow on the product space. If $X_{1}$ and $X_{2}$ are local flows, then the product $X_{1} \times X_{2}$ is a local flow, and if $S_{1} \subset X_{1}$ and $S_{2} \subset X_{2}$ are isolated invariant sets, then their product $S_{1} \times S_{2}$ is an isolated invariant set in the product flow.

LeMMA 5.1 (see [1]). If $\left(N_{1}, N_{0}\right)$ is an index pair for $S_{1}$ and $\left(N_{1}^{\prime}, N_{0}^{\prime}\right)$ is an index pair for $S_{2}$, then $\left(N_{1} \times N_{1}^{\prime}, N_{1} \times N_{0}^{\prime} \cup N_{1}^{\prime} \times N_{0}\right)$ is an index pair for $S_{1} \times S_{2}$. 
The following two lemmas are immediate.

LemMA 5.2. If $\left\{M_{\pi} \mid \pi \in(P,<)\right\}$ is a Morse decomposition of $S_{1}$, then

$$
\left\{M_{\pi} \times S_{2} \mid \pi \in(P,<)\right\}
$$

is a Morse decomposition of $S_{1} \times S_{2}$.

LEMMA 5.3. If $\{N(A) \mid A \in \mathscr{A}(P)\}$ is an index filtration for the Morse decomposition $\left\{M_{\pi} \mid \pi \in(P,<)\right\}$ of $S_{1}$, and $\left(N_{1}^{\prime}, N_{0}^{\prime}\right)$ is an index pair for $S_{2}$, then

$$
\left\{N(A) \times N_{1}^{\prime} \cup N(P) \times N_{0}^{\prime}\right\}
$$

is an index filtration for $\left\{M_{\pi} \times S_{2} \mid \pi \in(P,<)\right\}$.

We return now to the set-up of $\S 3$. Let $\left\{M_{\pi}^{0} \mid \pi \in\left(P,<_{0}\right)\right\}$ be a Morse decomposition of $S^{0}$ and $\left\{M_{\rho}^{1} \mid \rho \in\left(R,<_{1}\right)\right\}$ be a Morse decomposition of $S^{1}$. Then $\left\{M_{\pi}^{0} \mid \pi \in P\right\} \cup$ $\left\{M_{\rho}^{1} \mid \rho \in R\right\}$ is a Morse decomposition of $\hat{S}$. A connection matrix $\Delta$ for $\hat{S}$ with this Morse decomposition in the flow-defined order has the form

$$
\Delta=\left[\begin{array}{ll}
A & X \\
0 & B
\end{array}\right],
$$

where $A$ represents the $\Delta\left(\pi^{\prime}, \pi\right)$ entries, $B$ represents the $\Delta\left(\rho^{\prime}, \rho\right)$ entries and $X$ represents the $\Delta(\rho, \pi)$ entries, $\pi, \pi^{\prime} \in P$ and $\rho, \rho^{\prime} \in R$. Note that $(P, R)$ is an adjacent pair of intervals in the flow-defined order on $P \cup R$. In particular, $P$ and $R$ are intervals so $A$ and $B$ are upper triangular boundary maps.

THEOREM 5.4. A is the $\theta$-conjugate of a connection matrix for the Morse decomposition $\left\{M_{\pi} \mid \pi \in\left(P,<_{0}\right)\right\}$ of $S^{0}$ and $B$ is the $\theta$-conjugate of a connection matrix for the Morse decomposition $\left\{M_{\rho} \mid \rho \in\left(R,<_{1}\right)\right\}$ of $S^{1}$. In the first case $\theta$ has degree 0 ; in the second case $\theta$ has degree 1.

Proof. Each $M_{\pi}^{0}$ is isolated by a neighbourhood of the form $K \times[-\varepsilon, \varepsilon]$ as in lemma 3.3. It follows that for $\varepsilon$ small enough, each $K \times[-\varepsilon, \varepsilon]$ will remain an isolating neighbourhood if we deform the flow to the flow generated by

$$
\dot{x}=f(x, 0), \quad \dot{\lambda}=g(\lambda)
$$

in the obvious way. By remark 4.18 and theorem 4.22 , the restriction of the connection matrix to $\left\{M_{\pi}^{0} \mid \pi \in\left(P,<_{0}\right)\right\}$ in $\Phi_{t}$ is conjugate to a $\left\{M_{\pi}^{0} \mid \pi \in\left(P,<_{0}\right)\right\}$ in the product flow by a conjugation of degree 0 . It remains to show that any restriction $\bar{\Delta}(P)$ for $\left\{M_{\pi}^{0} \mid \pi \in P\right\}$ in the product flow is conjugate to a connection matrix for the Morse decomposition of $S^{0} \subset \mathbf{R}^{d}$. Let $\left\{N(A) \mid A \in \mathscr{A}\left(P,<_{0}\right)\right\}$ be an index filtration for $\left\{M_{\pi} \mid \pi \in P\right\} . \quad([-\varepsilon, \varepsilon], \varnothing)$ is an index pair for $\{0\} \subset \Lambda$. By lemma 5.3, $\{N(A) \times[-\varepsilon, \varepsilon] \mid A \in \mathscr{A}(P)\}$ is an index filtration for $\left\{M_{\pi}^{0} \mid \pi \in P\right\}$ in the product flow. The inclusion maps

$$
N(A) \rightarrow N(A) \times[-\varepsilon, \varepsilon], \quad x \mapsto(x, 0)
$$

induce chain equivalences on the quotients. It follows that the following diagram commutes for adjacent pairs $(I, J)$ :

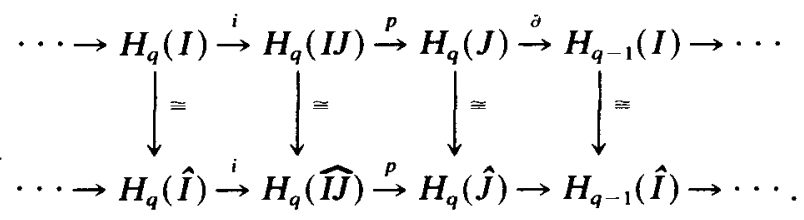


Here $H(I)$ denotes the homology of $h(M(I))$ in $\left\{M_{\pi} \mid \pi \in\left(P,<_{0}\right)\right\}$ and $H(\hat{I})$ denotes the homology of $h(M(I) \times\{0\})$ in the product flow. The vertical arrows are the inclusion-induced isomorphisms. Applying theorem 4.20 and remark 4.18, we see that $\bar{\Delta}(I)$ is conjugate to a connection matrix for $\left\{M_{\pi} \mid \pi \in P\right\}$.

For $\lambda=1$ we continue as above to

$$
\dot{x}=f(x, 1), \quad \dot{\lambda}=g(\lambda) .
$$

Let $\left\{N(A) \mid A \in \mathscr{A}\left(R,<_{1}\right)\right\}$ be an index filtration for $\left\{M_{\rho} \mid \rho \in\left(R,<_{1}\right)\right\}$.

$$
([1-\varepsilon, 1+\varepsilon],\{1 \pm \varepsilon\})
$$

is an index filtration for $\{1\} \subset \Lambda$. So $\{N(A) \times[1-\varepsilon, 1+\varepsilon] \cup N(P) \times\{1 \pm \varepsilon\} \mid A \in$ $\mathscr{A}(R)\}$ is an index filtration for $\left\{M_{\rho}^{1} \mid \rho \in R\right\}$ in the product flow. For $\left(A_{1}, A_{2}\right)$ an index pair in $\mathscr{A}\left(R,<_{1}\right)$ we have

$$
\begin{aligned}
& \frac{N\left(A_{2}\right) \times[1-\varepsilon, 1+\varepsilon] \cup N(P) \times\{1 \pm \varepsilon\}}{N\left(A_{1}\right) \times[1-\varepsilon, 1+\varepsilon] \cup N(P) \times\{1 \pm \varepsilon\}} \\
& \quad=\frac{N\left(A_{2}\right) \times[1-\varepsilon, 1+\varepsilon]}{N\left(A_{1}\right) \times[1-\varepsilon, 1+\varepsilon] \cup N\left(A_{2}\right) \times\{1 \pm \varepsilon\}} \\
& \quad=\sum\left(N\left(A_{2}\right) / N\left(A_{1}\right)\right) \text { the suspension of } N\left(A_{2}\right) / N\left(A_{1}\right) .
\end{aligned}
$$

From the Mayer-Vietoris sequence (see [3]) there is a natural isomorphism

$$
\psi: H_{q+1}\left(\sum\left(N\left(A_{2}\right) / N\left(A_{1}\right)\right)\right) \rightarrow H_{q}\left(N\left(A_{2}\right) / N\left(A_{1}\right)\right) .
$$

The naturality of $\psi$ implies that the following commutes for all adjacent pairs $(I, J)$ :

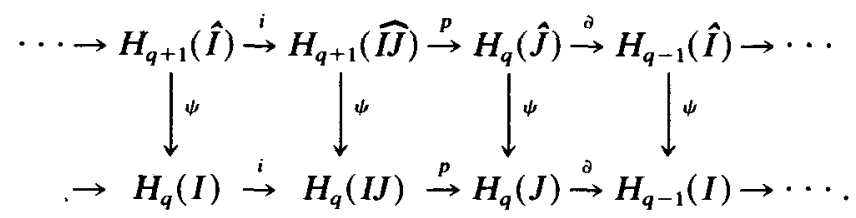

Here $H(\hat{I})$ means $H(h(M(I) \times\{1\}))$, and $H(I)$ means the homology of the index of $M(I) \subset \mathbf{R}^{d}$. By taking $\theta=\psi^{-1}$ and applying theorem 4.20 and remark 4.18, we conclude that $B$ is conjugate to a connection matrix for the Morse decomposition $\left\{M_{\rho} \mid \rho \in\left(R,<_{1}\right)\right\}$ of $S^{\mathrm{I}} \subset \mathbf{R}^{d}$, and since $\psi$ has degree $-1, \theta$ has degree +1 .

Definition 5.5. If $\Delta$ is a connection matrix for the flow $\Phi_{t}$,

$$
\Delta=\left[\begin{array}{ll}
A & X \\
0 & B
\end{array}\right],
$$

with $A$ and $B$ as in theorem 5.4, then $X$ is called the transition matrix for the flow generated by (3.1).

The reason for the term transition is as follows. Suppose we have a Morse decomposition which consists of hyperbolic rest points, and that the flows at $\lambda=0$ and $\lambda=1$ satisfy the transversality condition. These flows represent 'stable' states. $X$ contains information about unstable connections (e.g. connections between saddle points) as the system is deformed from one stable state to another.

The transition matrix contains information about connections from $M_{\rho}^{1}$ to $M_{\pi}^{0}$, so it can be used in conjunction with theorem 3.13 to detect connections at various parameter values. Since $\Delta^{2}=0$, we have $A X+X B=0$. 
LEMMA 5.6. $\operatorname{ker} \Delta=\operatorname{rank} \Delta$.

Proof. $h(\hat{S})=\overline{0}$, the pointed one-point space. This is most easily seen by continuing $g$ to a constant positive function (e.g. move the rest points together and 'cancel' them). This can be done in a way that preserves $N \times[-\delta, 1+\delta]$ as an isolating neighbourhood. It follows that $h(\hat{S})=h(\varnothing)=\overline{0} . H(\hat{S})=0$, so $H \Delta(\hat{S})=0$, which implies that $\operatorname{ker} \Delta=\operatorname{rank} \Delta$.

LEMMA 5.7. Suppose $\left(A, A^{*}\right)$ is an attractor-repeller pair in the isolated invariant set $S$. If $h(S)=\overline{0}$, then the flow-defined map $\partial: H\left(A^{*}\right) \rightarrow H(A)$ is an isomorphism.

Proof. Put $H(S)=0$ in sequence (4.8).

In some cases we can compute the entries of $X$.

Proposition 5.8. Suppose $M_{\pi}^{\lambda}$ continues for all $\lambda$ and suppose there is a compact set $U \subset \mathbf{R}^{d}$ such that for all $\lambda \in[-\delta, 1+\delta], U^{\lambda}$ is an isolating neighbourhood for $M_{\pi}^{\lambda}$, and for each $(x, \lambda) \in \partial U \times\{\lambda\}$ there is an $\varepsilon>0$ with $\Phi_{t}^{\infty}(x, \lambda) \in$ int $(U) \times\{\lambda\}$ for all $t \in[0, \varepsilon]$. Then if $\|g\|$ is small enough, the transition matrix entry $\Delta\left(M_{\pi}^{1}, M_{\pi}^{0}\right)$ is an isomorphism. Similarly, if $U^{\lambda}$ is an isolating neighbourhood for all $\lambda$, and for each boundary point $(x, \lambda) \in \partial U \times\{\lambda\}$ there is an $\varepsilon>0$ with $\Phi_{\imath}^{\infty}(x, \lambda) \notin U^{\lambda}$ for all $t \in[0, \varepsilon]$, then $\Delta\left(M_{n}^{1}, M_{\pi}^{0}\right)$ is an isomorphism.

Proof. If each boundary point of $U^{\lambda}$ flows into the interior (or exterior) of $U^{\lambda}$ under $\Phi_{t}^{\infty}$, it follows that each boundary point of $U \times(-\delta, 1+\delta)$ with $\lambda \in(-\delta, 1+\delta)$ flows into the interior (or exterior) under $\Phi_{t}$ if $\|g\|$ is small enough. It follows that $U \times[-\delta, 1+\delta]$ is an isolating neighbourhood for $M_{\pi}^{1} \cup M_{\pi}^{0} \cup C\left(M_{\pi}^{1}, M_{\pi}^{0}\right)$ if $\|g\|$ is small enough. By the properties of $U$, no Morse set lies between $M_{\pi}^{1}$ and $M_{\pi}^{0}$ in the flow-defined order on $P \cup R$, so the map $\Delta\left(M_{\pi}^{1}, M_{\pi}^{0}\right)$ is flow-defined. It is easy to verify that $(U \times[-\delta, 1+\delta], U \times\{1+\delta\})$ is an index pair if $\|g\|$ is small enough. It follows that $h\left(M_{\pi}^{1} \cup M_{\pi}^{0} \cup C\left(M_{\pi}^{1}, M_{\pi}^{0}\right)=\overline{0}\right.$, so $\Delta\left(M_{\pi}^{1}, M_{\pi}^{0}\right)$ is an isomorphism by lemma 5.7 .

Example 5.9. Consider the flows in figure 1. $S^{\lambda}$ is the maximal invariant set in a rectangle $N$. The rest points $M_{1}, M_{2}$ and $M_{3}$ form a Morse decomposition for $S^{0}$
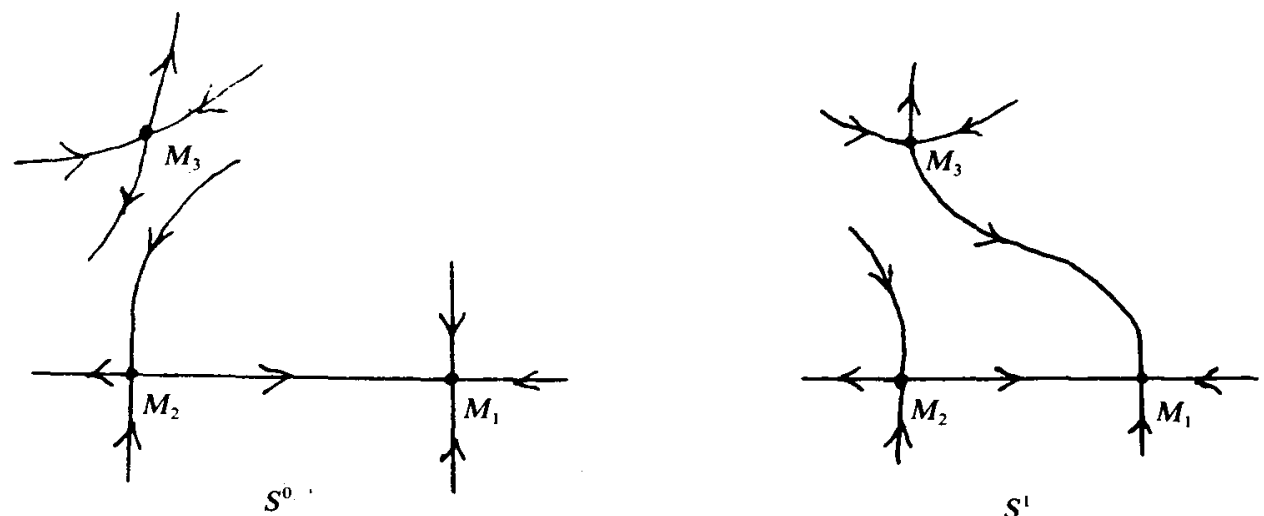

FIGURE 1 
and $S^{1}$. We assume that $N$ remains an isolating neighbourhood and that the rest points continue as a Morse decomposition for each $\lambda . h\left(M_{1}\right)=\Sigma^{0}$, the pointed 0 -sphere, and $h\left(M_{2}\right)=h\left(M_{3}\right)=\Sigma^{1}$, the pointed 1-sphere. In both $S^{0}$ and $S^{1}, h\left(M_{1} \cup\right.$ $\left.M_{2} \cup C\left(M_{2}, M_{1}\right)\right)=\overline{0}$, so the map $\Delta(2,1)$ is an isomorphism in both cases. Let $\Delta_{0}$ denote the connection matrix for $S^{0}$ and $\Delta_{1}$ the connection matrix for $S^{1}$. In $S^{0}$, $\Delta_{0}(3,1)=0$ since $C\left(M_{3}, M_{1}\right)=\varnothing$. In $S^{1}, h\left(M_{1} \cup M_{3} \cup C\left(M_{3}, M_{1}\right)\right)=\overline{0}, 3$ and 1 are adjacent in the flow-defined order, so $\Delta_{1}(3,1)$ is an isomorphism. $\Delta(2,1)=0$ in both cases by degree considerations. Thus we have

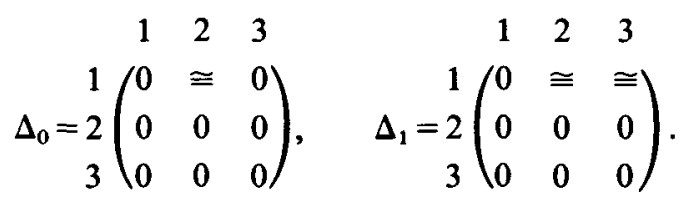

$\Delta_{0}$ and $\Delta_{1}$ are unchanged by conjugation since $H(\pi)$ is one-dimensional. We consider $\Delta$, the connection matrix for the flow $\Phi_{t}$. By lemma 5.8 , the entry $\Delta\left(M_{1}^{1}, M_{1}^{0}\right)$ is an isomorphism. We have

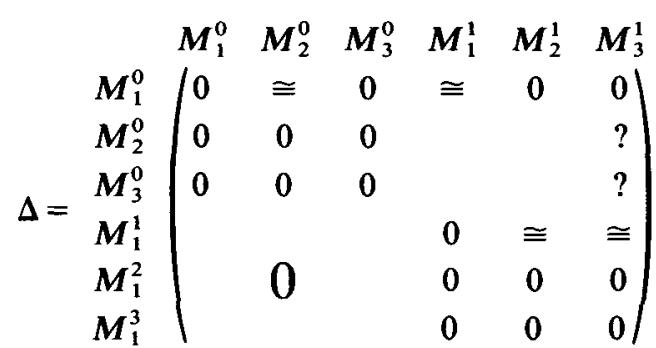

and $\Delta^{2}=0$. The zeros in the top row come from degree considerations. $h\left(M_{3}^{1}\right)=$ $h\left(M_{2}^{1}\right)=\Sigma^{2}$ and $h\left(M_{1}^{0}\right)=\Sigma^{0}$, so there is no degree -1 map on homology. Multiplying the top row and the last column, we see that $\Delta\left(M_{3}^{1}, M_{2}^{0}\right) \neq 0$ for all $g$. Thus $M_{2}^{0}<M_{3}^{1}$ in the flow-defined order. It is easy to verify that this implies $C\left(M_{3}^{1}, M_{2}^{0}\right) \neq \varnothing$ for any g. We now let $\|g\| \rightarrow 0$ and apply theorem 3.13. Since $M_{1}^{\lambda}$ is an attractor for all values of $\lambda$, we conclude that there is a $\lambda \in(0,1)$ with $C\left(M_{3}^{\lambda}, M_{2}^{\lambda}\right) \neq \varnothing$, i.e. the saddles are connected for some parameter value.

Further applications of the theory are discussed in a paper by Mischaikow [8] and will be discussed in a future paper by the present author.

Acknowledgements. Some of the results of this paper are from the author's thesis, written under Charles Conley. I would like to thank Charlie for his help and guidance. His wealth of ideas, enthusiasm and sense of humour made discussions with him profitable and enjoyable. I will miss him very much. I would also like to thank Dick McGehee, Rick Moeckel and Don Aronson for their help with the completion of my thesis.

\section{REFERENCES}

[1] C. C. Conley. Isolated Invariant Sets and the Morse Index. AMS Regional Conference Series in Mathematics 38. AMS, Providence, RI (1978). 
[2] C. C. Conley \& E. Zehnder. Morse-type index theory for flows and periodic solutions for Hamiltonian equations. Comm. Pure Appl. Math. 37 (1984), 207-253.

[3] A. Dold. Lectures on Algebraic Topology. Springer-Verlag, New York (1972).

[4] R. Franzosa. Index filtrations and connection matrices for partially ordered Morse decompositions. Thesis. University of Wisconsin, Madison (1984).

[5] R. Franzosa. Index filtrations and the homology index braid for partially ordered Morse decompositions. Trans. AMS (to appear).

[6] R. Franzosa. The connection matrix theory for Morse decompositions. Preprint.

[7] H. Kurland. Homotopy invariants of a repeller-attractor pair, I: The Puppe-sequence of an R-A pair. J. Differential Equations 46 (1982), 1-31.

[8] K. Mischaikow. Classification of travelling wave solutions of reaction-diffusion equations. Preprint.

[9] J. Munkres. Topology: A First Course. Prentice-Hall, Englewood Clifis, NJ (1975).

[10] J. Reineck. The connection matrix and the classification of flows arising from ecological models. Thesis. University of Wisconsin, Madison (1985).

[11] J. Robbin \& D. Salamon. Dynamical systems, shape theory and the Conley index. Ergod. Th. \& Dynam. Sys. 8* (1988), 375-393.

[12] D. Salamon. Connected simple systems and the Conley index of isolated invariant sets. Trans. AMS 29 (1985), 1-41. 